\title{
Oxford Handbook for the Philosophy of Science
}

\author{
Paul Humphreys \\ University of Virginia
}

\section{OXFORD}





\title{
1 \\ Philosophy of the Physical Sciences
}

\author{
Carl Hoefer \\ Chris Smeenk
}

\begin{abstract}
We survey some debates about the nature and structure of physical theories and about the connections between our physical theories and naturalized metaphysics. Our discussion is organized around an "ideal view" of physical theories, and criticisms that can be raised against it. This view includes controversial commitments regarding the best analysis of physical modalities and inter-theory relations. We consider the case in favor of taking laws as the primary modal notion, discussing objections related to alleged violations of the laws, the apparent need to appeal to causality, and the status of probability. The "ideal view" includes a commitment that fundamental physical theories are explanatorily sufficient. We discuss several challenges to recovering the manifest image from fundamental physics, along with a distinct challenge to reductionism based on acknowledging the contributions of less fundamental theories in physical explanations.

Keywords: modality, laws, causality, reductionism, probability, inter-theory relations
\end{abstract}

\subsection{Introduction}

Understanding the nature of our knowledge of physics has long been a central topic in philosophy of science. The introduction of relativity theory and quantum mechanics in the early twentieth century inspired the likes of Reichenbach and Carnap to develop distinctive accounts of epistemology and the structure of theories. Their views about scientific knowledge and later quite different views have often shared one feature, namely taking physics as exemplary. We applaud the recent reversal of this trend, as philosophers considering other areas of science have set aside the physical sciences as a model. Yet there are a variety of philosophical issues that are closely intertwined with the physical sciences. We will briefly survey here some aspects of the connection between physics and naturalized metaphysics, starting from a "received view" of the nature and scope of physical theories, and exploring how challenges to that view may have ramifications for the philosophical consequences of physics.

\subsection{The Ideal View}

A number of philosophical issues can be thought of as arising in and for the totality of the physical sciences: issues having to do with their aims, the structure of their 


\section{Philosophy of the Physical Sciences}

theories, their presupposed world-view, and the relationship between different theories. To introduce these issues, we will begin with a simplified account of the nature of physical theories, illustrated by point-particle mechanics. Drawing on this sketch of a physical theory, we will state an overall position which we will call the Ideal View ('IV' for short). ${ }^{1}$ Roughly speaking, this view is a mixture of fundamentalism, scientific realism, and optimism about the simplicity of nature. We expect that certain aspects of the view, or suitably refined versions of it, will appeal to many philosophers of the physical sciences. Yet each of its claims has been challenged in recent philosophical discussions. In ensuing sections, we will review these challenges and assess the prospects for defending the view.

The image of the physical world as having a simple ontology of matter in motion, governed by unchanging laws, comes from classical mechanics. The simplest version of mechanics describes the dynamical evolution of a system of point particles due to forces acting among them. The kinematics of the theory specifies the geometry of motion and the kinds of particles under consideration, whereas the dynamics fixes the forces and their effects. In the Hamiltonian formulation, the state of the system at a given time is represented by a point in phase space $\Gamma$, specifying the position and momenta for each of the particles relative to a given reference frame. The inter-particle forces are captured in a single function called the Hamiltonian, which determines how the particles move via Hamilton's equations. The evolution of the system over time is a trajectory through $\Gamma$; this specifies the history of the system. The semantics of the theory depends on linking this theoretical description to (idealized) experimental situations. At least in principle, such a link establishes how regions of phase space correspond to answers to experimental questions, such as "The value of property $\mathrm{P}$ is given by $X \pm \Delta$ (in appropriate units)". One central project of philosophy of physics is to interpret physical theories in the sense of specifying such semantic links; or, more succinctly, to understand how real physical systems should behave if the theory were true.

This sketch is enough to formulate our foil, a set of four commitments making up the IV. Although the details vary considerably for other physical theories, the structure described above for Hamiltonian mechanics is quite general: many other theories also introduce basic types of entities and their possible states, in concert with modal commitments characterized by laws. In the case of Hamiltonian mechanics, the basic ontology includes particles (possibly of distinct types) and the spacetime through which they move, and Hamilton's equations give the dynamics. The IV adds the following philosophical commitments, and aspirations regarding the progress of physics, to this account of the structure of theories:

- Ontology: theories postulate a set of basic entities, which are the building blocks of all entities falling within the domain of the theory.

- Laws: the dynamical laws governing the behavior of the basic entities have nontrivial and non-subjective modal force and explanatory power, and they do not admit exceptions. The laws may either be deterministic, in the sense that a given

\footnotetext{
${ }^{1}$ The ambiguity in 'ideal' - between 'perfect, excellent,' as opposed to 'imaginary, not real or practical' - is intentional, and reflects the contrasting assessments of the co-authors.
} 
physical state uniquely fixes a dynamical history, or stochastic, assigning a probability distribution over possible histories. Laws have privilege over other modal notions such as: cause, disposition, power, and propensity; this means, among other things, that those latter notions need not be invoked in a deep or ineliminable way in presenting or interpreting fundamental physical theories.

- Fundamentalism: there is a partial ordering of physical theories with respect to "fundamentality." The ontology and laws of more fundamental theories constrain those of less fundamental theories; more specifically: (i) the entities of a less fundamental theory $T_{i}$ must be in an appropriate sense "composed out of" the entities of a more fundamental theory $T_{f}$, and they behave in accord with the $T_{f}$ laws; (ii) $T_{f}$ constrains $T_{i}$, in the sense that the novel features of $T_{i}$ with respect to $T_{f}$, either in terms of entities or laws, play no role in its empirical or explanatory success, and the novel features of $T_{i}$ can be accounted for as approximations or errors from the vantage point of $\mathrm{T}_{\mathrm{f}}$.

- Status of a Final Theory: finding the "final theory," which identifies the fundamental entities and laws and is, in some sense, the ultimate source of physical explanations, is an appropriate aim for physics.

Together these claims constitute a controversial view about the relationship between physical theories and naturalized metaphysics, and about the structure of physical theories. Debates about this view run like a rich vein through much recent work in philosophy of the physical sciences, which we will mine for insights in the sections below.

But first we would like to clarify the view in relation to two issues. First, the constraints $T_{f}$ imposes on $T_{i}$ according to fundamentalism need not be as restrictive as philosophers often suppose. Fundamentalists, as we have formulated the view, can freely acknowledge that less fundamental theories may have enormous pragmatic value due to their computational tractatibility, simplicity, and other features. They may also play a crucial role in interpreting more fundamental theories. The constraints imposed rather concern ontology and modality. The empirical and explanatory success of $T_{i}$ must be grounded in the fact that it captures important facts about the deeper structures identified by $T_{f}$. Or, in other words, $T_{i}$ 's successes should be recoverable, perhaps as a limiting case within a restricted domain, in terms of $T_{f}$ 's ontology and laws. A second reason why the consistency requirement is weak is because physics is promiscuous when it comes to physical properties. In Hamiltonian mechanics, for example, essentially any mapping from phase space to real numbers qualifies as a "physical property" that can be ascribed to a system. There is no requirement that legitimate properties have a simple definitional link to the basic entities used in setting up the theory. As Wilson (1985) emphasized, the generality with which physics handles properties has been essential to its successful treatment of higher-level quantities such as "temperature." 2 Many philosophical treatments of the relationship between the

\footnotetext{
${ }^{2}$ Contrary to conventional wisdom among philosophers, temperature is not simply identified with "mean molecular kinetic energy." That relationship only holds in a quite limited case, namely an ideal gas; for gases with non-negligible interactions, not to mention liquids or solids, the mean kinetic energy depends on temperature and other physical parameters. This complexity blocks a simple "definition" of temperature, but poses no obstacle to treating temperature as a physical property.
} 


\section{Philosophy of the Physical Sciences}

"special sciences" and physics overlook the importance of this point, imposing a more tightly constrained relationship between higher-level and physical properties than that used in physics itself. Fundamentalists of different persuasions are of course free to introduce and defend different accounts of inter-theory relationships, but we do not see stronger versions as following directly from the practice of physics.

Second, the sense in which laws "govern behavior" has to be treated with some care, in order to defend the view that the laws do not admit exceptions. A naïve account of laws ties their content directly to behaviors manifested by various systems falling within their domain of applicability - the law of gravity governs the falling of an apple, for example. Yet such systems often manifest behavior that apparently runs counter to these same laws. Does a leaf falling quite differently than the apple somehow render the law of gravity false? The idea that manifest behaviors straightforwardly falsify laws goes wrong in too closely assimilating the content of the laws with that of equations of motion derived from the laws (see, in particular, Smith 2002). ${ }^{3}$ The laws in conjunction with a variety of other ingredients, such as fluid resistance in a model of projectile motion, are used to derive specific equations of motion. These other ingredients needed to apply the theory to concrete situations do not have the modal force or status of the laws, and neither do the derived equations of motion. The derived equations for the apple may fail to apply to the leaf, yet that does not show that the laws of mechanics are "false" or admit exceptions. The laws of all existing theories admit exceptions in a very different sense, namely that there are phenomena falling within overlapping domains of applicability of theories which have not yet been successfully combined. The fundamentalist assumes, however, that it is reasonable to aim for a "final phyiscs" that applies universally, and it is the laws of this theory that truly admit no exceptions.

\subsection{Interpretation}

Philosophers have often taken up the challenge of interpreting physical theories, in the sense of explaining what the world would be like if the theory were true (see, e.g., van Fraassen 1989). This project is notoriously challenging in the case of quantum mechanics, for which there is still no satisfying interpretation despite its empirical success, but a variety of interpretative questions arise for other physical theories as well. An interpretation should characterize the physical possibilities allowed by the theory, as well as specifying how the mathematical structures used by the theory acquire empirical content. The standard account of the interpretative project fits well with the IV: the laws of the theory delimit the space of physical possibilities, perhaps regarded as the set of worlds that are physically possible according to the theory. The differences among these possible worlds reflect brute contingencies, such as details about the initial state of the universe. The interpretative project then attempts to explain what these possible worlds would be like, based on the general features of the theory (such as its basic ontology, kinematics, and dynamics). Here we will briefly explore a recent line of criticism of this approach to interpretation, which argues that

\footnotetext{
${ }^{3}$ Physicists' usage of the term "laws" can confuse the issue; various derived equations that are commonly called "laws" don't deserve the honorific, on our view.
} 
interpretations pursued at this abstract level fails to account for the explanatory power and other virtues of our theories.

An interpretation in the traditional sense starts from the general structure of the theory, as described above: the laws of the theory and its state space. Assigning empirical content to these structures often begins with appeals to preceding theories, whose interpretation is taken to be uncontroversial. To discuss one example briefly, the basic laws of general relativity (Einstein's field equations) determine what sort of gravitational field (= spacetime metric) is compatible with a given large-scale matter distribution (represented by the stress-energy tensor field), and vice versa. But these structures are only connectable to experiment and observation via principles associating the possible trajectories of different kinds of particles or test bodies (massive, massless, force-free) with different kinds of curves in a relativistic spacetime, and the proper time elapsed along a worldline to the time recorded by an ideal clock. (See Malament (2007) for a particularly clear presentation.) These principles clarify the physical content of the spacetime geometry introduced in GR provided an antecedent understanding of physical trajectories and classification of different types of bodies or particles. The connection between solutions of Einstein's equations and "experiment" or "observation" is mediated by earlier theories that relate data to the determination of a spacetime trajectory - for example, the theories involved in establishing the trajectory of a planet based on a set of astronomical observations.

One recent line of criticism focuses on whether the laws and state space of a theory are approriate starting points for the interpretative project. Ruetsche (2011), in particular, develops a pragmatist account of interpretation, according to which questions of possibility should be posed with respect to particular applications of the theory. Her arguments depend on recondite details of quantum mechanics applied to $\infty$-dimensional systems, but roughly put she focuses on cases in which the mathematical structures needed to support a schematic representation of a physical system cannot be introduced at the level of generality where philosophers operate. Suppose we require that any bona fide physically possible state of a system must have a well-defined dynamical evolution, specifying how it evolves over time. For some models (such as that of ferromagnetism in an infinite spin chain, discussed in her §12.3), implementing this criterion will depend on the dynamics one chooses - for some (otherwise kosher) states, the dynamics is not well-defined. The particular application one has in mind will determine which dynamics, and hence which states, should be preferred. Based on such cases, Ruetsche argues that interpretive questions need to be indexed to particular contexts of application of a theory rather than at an entirely general level. We expect that Ruetsche's line of argument applies more generally, but making the case would require a more detailed assessment of the parallels between her cases and examples from other domains of physics than we can pursue here.

\subsection{Modality}

A perennial problem for our understanding of physics is that of adequately describing and accounting for its modal aspects: physical necessity, physical possibility, and physical probability. In this section we will explore the dialectic of debates between two ways of approaching physical modalities: those which make primary the concept 


\section{Philosophy of the Physical Sciences}

of law of nature, and those which demote laws and make primary some other modally loaded notion such as cause, capacity, disposition or propensity. There is of course a long tradition in the history of natural philosophy of denying that there is any real necessity in physical processes at all, and arguing that the necessity we feel that they have is precisely that: merely felt necessity, a matter of our psychology and/or sociology rather than a feature of nature itself. Proponents of such views include Hume, Ayer, Goodman, van Fraassen and Marc Lange, among many others. ${ }^{4}$ We will not discuss this tradition, nor the Kantian tradition which seeks to give a transcendental or psychological grounding to modality in nature. Kantian projects simply fail at giving a modal grounding to the regular behavior in nature, while the subjectivist tradition does not even try to offer such a grounding. Both traditions also have had trouble, traditionally, to make sense of the modal aspects implicit in actual scientific practice.

\subsubsection{Laws of nature}

The idea that all physical processes occur in conformity with precise, mathematical regularities is one that has been a dominant assumption in Western science since the time of Galileo. But 'regularity' and 'conformity with' do not adequately capture the strength of the law-idea. Events do not just happen to respect the law-regularities; in some sense they have to, it is a matter of [what is usually called] "physical necessity". One way of cashing out that necessity, not widely held now, is to ground it in an omnipotent and omniscient law-giver (or law-enforcer).

Without appealing to the divine, it is hard to explain the necessity of natural laws. One way to go would be to equate physical necessity with metaphysical necessity: the supposed category of necessary truths that follow from the essences or natures of things. Metaphysical necessity is supposed to be non-logical in character, and how to understand and define it is also a difficult issue, making it a less than ideal resource for explaining or grounding physical necessity. But the basic idea has intuitive appeal: Things such as electrons, neutrinos or photons behave in the ways that they do because of their fundamental natures; if they behaved differently, we feel inclined to say, then they would not be the same kinds of things. A particle that attracted other electrons rather than repelling them would simply not be an electron; it would be a positron, perhaps, or some entirely different kind of thing. More generally, this line of thought holds that the universal truths physicists have discovered about fundamental particles and fields are precisely the truths that are, or follow from, their essential natures.

But many philosophers have other intuitions, equally or more strongly held, that go against the metaphysical necessity of laws. These are intuitions concerning the contingency of many physical facts, including many facts that appear in physical laws. A good example is the apparent contingency of the values of most or all natural constants (e.g. c, the speed of light $\left(2.998 \times 10^{8} \mathrm{~m} / \mathrm{s}\right)$; or $G$, the gravitational constant, $\left.\left(6.67384 \times 10^{-11} \mathrm{~m}^{3} \mathrm{~kg}^{-1} \mathrm{~s}^{-2}\right)\right)$. Physicists can give no good reason why the numerical values of these constants (or their ratios) could not have been slightly - or even greatly - different from what they actually are; and many philosophers would argue that this is for a very good reason: there is no such reason, these values are a matter of

\footnotetext{
${ }^{4}$ This tradition includes modern-day Humeanism of the variety championed by David Lewis; see Loewer (2004a) for an excellent overview.
} 
pure contingency. ${ }^{5}$ Other examples often cited in support of contingency involve the mathematical forms of the laws themselves: that the gravity equations have (or lack) a cosmological constant, that the laws of electromagnetism are Lorentz-covariant rather than Galileo-covariant, and so on. But the consensus in favor of contingency is by no means complete, and many physicists hope that in the light of future theories some of these apparent contingencies may turn out to be necessities given "deeper" level laws. What has never been made clear by even the most hopeful defender of the necessity of physical law, is how the necessity could be made complete, i.e., all contingency purged from fundamental physics. ${ }^{6}$

Coming back to the idea of physical necessity being a matter of the essential natures of the basic physical kinds, we note that there is more than a passing resemblance between this "explanation" of physical necessity and the 'virtus dormitiva' explanation of opium's soporific effects derided by Moliere. The explanation seems to be nothing more than a restatement, in slightly different words, of the claim of necessity. And if we are asked to spell out, for example, what the essential nature of an electron is, there is no way we could do so without invoking the mathematical laws of physics we have discovered so far, which are all we have available to - in part at least - capture the way electrons behave. We find ourselves going around in a very tight circle. Since the laws at least have precise mathematical contents, and (given auxiliary assumptions) precise and testable empirical consequences, it is no wonder that many philosophers prefer to stick with mathematical laws themselves and avoid talk of 'natures', or related modally-loaded notions such as power, disposition, tendency, cause. We will return to these related notions in the next subsection; for now let us proceed assuming that the laws of nature have a sui generis form of necessity, equivalent neither to brute contingency nor to metaphysical or logical necessity.

In addition to the difficulty of spelling out out the nature of natural necessity, laws have been challenged by philosophers who question whether the laws - those we have already found in physics - can even be said to be true, in a respectable sense of "approximately true". We set aside the fact that extant examples of physical laws all come with domain restrictions, e.g. for classical Newtonian mechanics the restriction to macroscopic bodies and relative speeds $\ll c$. Even within their intended domains, laws may seem to fail frequently. A pendulum bob loses momentum and eventually stops, even in a vacuum - due to friction at the axis. Newton's predictions for Mercury's perihelion fail by a little bit - because of general relativity, we might say,

\footnotetext{
${ }^{5}$ In cosmology, anthropic reasoning has at times been invoked to justify constraints on the range of values that certain constants could have (if the universe is to be one with observers). But such arguments, if accepted, merely narrow the range of values that the constants could have; the contingency of the actual values or ratios is still present.

${ }^{6}$ An idea worth mentioning in this connection is this: that the regular behavior we see in the physical world, and codify in the laws of physics, is in fact something that emerges from pure chaos or randomness at the most fundamental/microscopic level. This idea would give a kind of (probabilistic) relative necessity to the known laws of physics, but eliminate laws from the most fundamental level. Examples of how higher-level regularities can emerge from chaos or randomness at a lower level have been discussed in depth by Strevens (2003, 2011) and Myrvold (forthcoming) in the context of thermodynamics and statistical mechanics. Filomeno (2014) provides a thorough discussion of speculations by physicists (e.g. Froggatt \& Nielsen (1991), Wheeler (1982), Unger \& Smolin (2014)) regarding such a non-law-grounded emergence of the lawlike behavior codified in quantum theories. But these speculations remain sketchy and incomplete.
} 


\section{Philosophy of the Physical Sciences}

indicating that the earlier applications of Newton's theory extend beyond the domain in which it approximates general relativity. And many quantum systems treated with Schrödinger's equation fail to come out right unless we introduce apparently ad hoc terms into the Hamiltonian, unmotivated by fundamental theory. Nancy Cartwright is the philosopher who has most forcefully pushed the "laws are false" objection, but many agree with the thrust of her critique.

In the debate over laws' failure to be true initiated by Cartwright (1983), the problem is often presented in terms of physical laws' needing a ceteris paribus clause. The laws describe what will happen, ceteris paribus, i.e., as long as nothing interferes. Since it is typically impossible to fully cash out what is meant by 'ceteris paribus' for any given law, the defenders of laws typically reject the claim that there is such an intended clause appended to law statements. An alternative to accepting the implicit presence of a ceteris paribus clause is the perspective mentioned at the end of section 1.2: the derivation of empirical equations of motion from general laws is something we do using carefully chosen supplemental conditions (usually mathematically expressable) and having a restricted range of intended application. Outside that range, or when the supplemental conditions do not hold, the equations of motion will not work, but that is not a falsification of the more fundamental general laws.

What tends to be the case when prima facie law-failure cases are raised is that we are not faced simply with a failure of the law or laws to accurately describe things. Instead, scientists typically know how to explain the corrections that are needed, via de-idealizations of various kinds, introduction of causes from other theories or from direct observation (which can be mathematized appropriately so as to be plugged into the laws), etc. The defender of laws and fundamentalism sees here a non-troubling upshot of the complexity of nature, a complexity that the most fundamental laws can, she hopes, fully explain. We introduce an ad hoc friction force to get the pendulum's behavior right, for example, but friction is itself just a consequence of the fundamental quantum laws governing condensed matter.

But a different perspective is possible: one that emphasizes the near-ubiquity of the intrusion of talk of causes and effects in our application of physical laws to realworld systems, both inside and outside the laboratory. In addition to Cartwright, Mathias Frisch has forcefully argued for the importance and ineliminability of causal talk in physics; see Frisch (2009a) and (2012). Turning the tables on physical law, some philosophers argue that the notion of cause, and related notions such as power, disposition, or capacity, are conceptually basic and metaphysically prior, while the appearance of precise, mathematical law-governed regularity is nothing more than the upshot of the truism "same cause, same effect".

\subsubsection{Causes, powers, dispositions etc. $v s$ laws}

Russell (1912) famously argued that the notion of cause had no place in modern physics; like the monarchy, it was a relic of medieval times that had long outlived its utility. Much more recently John Norton has taken up the crusade against causation, in his (2007). Norton characterizes causation as a 'folk-science' concept that is certainly useful in daily life and in applications of physics, but which does not deserve to be thought of as part of the deep structure or content of nature as it is given to us by our 
physics theories. Mathias Frisch responded to Norton in defense of the ineliminability of causal talk from even fundamental physics (2009a), which led to further exchanges. ${ }^{7}$

While we think Frisch makes excellent points about the crucial role that causation seems to play at times in physics, there is arguably a fundamental explanatory asymmetry - alluded to already above - that favors the priority of laws over the other modal notions (cause, disposition, power etc.) and helps explain the enduring presence of laws and fundamentalism in the IV. The asymmetry is this: While causal talk seems unable to explain the utility and universality of laws in a non-shallow sense, universal and exceptionless laws do seem able to ground the utility of causal talk and explain why causes sometimes don't produce their normal effects in a non-shallow sense. Without translation into contentful mathematical statements, talk of causes, powers and so forth has at most a rough qualitative meaning, and the explanatory power is limited or zero. For example, consider the striking of a match and its subsequent burning.

One way to characterize this phenomenon is using disposition-talk. The matchhead material has a disposition to ignite when in the presence of sufficient heat (and oxygen); striking the match produces the needed heat via friction; so if all the necessary triggering conditions are present, and no counter-acting powers (e.g. falling raindrops or high winds), striking the match produces the effect of ignition. Another way to characterize the phenomenon would be by digging down into the quantum chemistry of the match-head material and treating the combustion phenomenon (chemical reactions binding oxygen and releasing energy in the form of molecular motion, i.e., heat) using mathematical equations and models. Many philosophers would argue that the latter sort of characterization is more explanatory than the former. ${ }^{8}$

The point, again, is that explanatory power may constitute a real asymmetry between laws and [talk of] causes, powers or dispositions. If we wish we can talk about what electrons and atoms do using the language of powers and dispositions. But doing so seems to provide only a thin gloss of familiarity, and does not come close to providing the detailed predictive power that models constructed from the laws can have. The advocates of causality and powers would surely agree with this, but add that they have every right to help themselves to the mathematical laws and models of physics in formulating their explanations, because those laws simply express in precise terms the causal upshot (in certain circumstances) of the causal powers and dispositions of things.

Here the defender of fundamental laws will object that it is the mathematical laws per se that do the real explanatory work, and many of them cannot plausibly be read as statements about causal powers and so forth. It is fine to say that, for example, He nuclei have the capacity to bind with two electrons, and that those electrons are disposed to occupy only certain discrete energy states. But this gives us no handle on when and why the bonds are stable, what the allowed energy levels are, the shapes of the orbitals,

\footnotetext{
${ }^{7}$ See Frisch (2009b) and Norton (2009) for this exchange. Smith (2013) defends a neo-Russellian view, considering various cases that Frisch (and others) see as reflecting causal commitments. Smith argues that the conditions thought to reflect causality principles are more aptly regarded as motivated by other concerns, such as imposing conditions needed to insure the existence and uniqueness of solutions to a given equation.

${ }^{8}$ See Hoefer (2003) for a separate line of argument in favor of fundamental laws. For a general discussion of reduction, see the chapter by Hütteman \& Love in this volume.
} 
and so forth; the Schrödinger equation does give us these things. And the fundamentalist will argue that the Schrödinger equation, and Einstein's field equations, and Maxwell's equations and perhaps other fundamental laws, cannot be plausibly read as causal power/disposition/capacity statements, nor as upshots or consequences of such statements. In order for these laws to be consequences of some (explanatorily) more fundamental causal power/capacity/disposition statements, the latter would have to be expressed in mathematical language, yet still clearly be expressions that can be given a causal gloss (as is possible for certain laws like Newton's law of gravity and Coulomb's law). But we have no examples of any such more-fundamental statements that could ground our most fundamental laws such as the Schrödinger equation. So the law fundamentalist sees an explanatory asymmetry in favor of mathematical laws over causes, powers, capacities and dispositions.

Authors such as Cartwright, Frisch, and Woodward (2003) have argued very persuasively that talk of causation, and related Aristotelian notions, is prevalent throughout the physical sciences, both in practical applications and in theoretical discussions. The priority that the IV gives to laws of nature can be partly defended, but it remains very controversial.

\subsubsection{Probabilistic law}

A question of perennial interest in the physical sciences is that of determinism: do natural systems always evolve in the same way, if they start from exactly the same initial conditions? With the rise of classical mechanics and its influence on philosophy in the modern period (from Descartes to Kant), it was common to assume the truth of determinism until the phenomena of quantum mechanics appeared to inject intrinsic randomness into nature. In the dominant standard interpretation of QM (and quantum field theories), the theory makes only probabilistic predictions in many situations. Via the 'Born Rule', the state vector of a quantum system prescribes probabilities for certain events to occur. Taken together, the laws and rules of quantum physics again, as standardly presented and interpreted - appear to be essentially stochastic.

In our laying out of the IV above we incorporated stochastic laws explicitly as one possible type of physical law, and this is typically what defenders of fundamental laws have done. But it should be noted that some physicists and some philosophers are not happy with the notion of irreducible stochastic laws. There are at least two sorts of root for the discontent. One is simply an aversion to indeterminism: for many, it is hard to give up on the truth of the Principle of Sufficient Reason in some form or other. When things happen, the intuition goes, there is always a reason why they happened thusly, and if we have at the moment only statistical laws to describe these events, that is a defect in our physics that we should seek to correct. ${ }^{9}$ Einstein was

\footnotetext{
${ }^{9}$ While some philosophers and physicists argue against the notion of (irreducibly) chancy fundamental laws, interestingly, a number of physicists have recently argued that experiments establishing violation of Bell inequalities by quantum systems prove the existence of intrinsic randomness in nature (e.g. Colbeck \& Renner (2012), Pironi, Acín et al (2010)).

Using reasoning similar to that of Bell in his famous 1964 theorem, these authors argue that when pairs of space-like separated events display correlations violating a Bell inequality, then we can exclude the possibility that there is some underlying deterministic account of the correlations, thus leaving pure stochasticity as the only option. Their arguments depend however on assumptions that exclude
} 
one physicist (/philosopher) who took a dim view of stochastic laws for this sort of reason, but there are many others. ${ }^{10}$

A second root of discontent with stochastic laws has to do with puzzlement about their epistemic status and their truth-conditions. ${ }^{11}$ Suppose we have a stochastic (fundamental) law that entails that the chance of $R_{i}$ being the outcome in experimental setup $S$ is $x_{i}=0.343$. What does this entail about what will happen in the world? This is a question that may appear to have no good answer, because - strictly speaking, and using the understanding of this chance as a primitive fact - any actual frequency of $R_{i^{-}}$ outcomes you care to name is physically possible. And in this sense the content of the law is no different than that of an alternative law that sets $x_{i}=0.395$, or $x_{i}=0.840$. So we have a prima facie puzzle about the semantic content of the stochastic law statement. Now, as we all know, if the law says $x_{i}=0.343$, then that entails that in a set of many $S$-experiments the frequency of $\mathrm{R}_{i}$-outcomes is unlikely to be very different from 0.343 , and far more unlikely to be close to 0.840 than to 0.395 . These claims about what is 'likely' or 'unlikely' are, of course, themselves probability claims; and if we arrive at them by simply assuming independence of our S-experiments and applying the axioms of probability, then these claims are themselves just claims about irreducible objective chances. If we started out wondering what the semantic content of such claims might be, being offered further such claims in response is obviously no help.

The reason why this problem is not more often noted is that when we make our claims about what the outcome-frequencies are likely or unlikely to be, we tacitly invoke the "Principal Principle" (PP), turning the objective chances of certain frequencies' obtaining into a subjective probability - i.e., a credence, or level of expectation, or betting ratio. ${ }^{12}$ In so doing we inject some cash value into the claims, because (for example) we do things like rejecting hypotheses that have less than $1 \%$ or $5 \%$ subjective probability. This is the basis of classical statistical testing. So, for example, if our calculations lead us to have subjective probability of less than $1 \%$ that the objective chance of $R_{i}$ is different from $x_{i}(=0.343)$ by more than 0.05 , given the outcomes we have observed, then we reject all possible values for $x_{i}$ greater than 0.393 or less than 0.293 .13

So we feel both that we know what the content of our objective probability claims is, and how to test them. But the crucial move was our use of the Principal Principle to turn irreducible chance-facts into rational subjective degrees of belief. And it is

certain kinds of possible deterministic "hidden variable" alternatives to QM, including theories like Bohmian mechanics, on the basis of the type of non-locality and contextuality such theories must involve, and many philosophers would find these assumptions too strong to be defensible.

${ }^{10}$ As Arthur Fine (1986) and others have shown, Einstein was more concerned about the "spooky action at a distance" of QM than about its indeterminism. But there are many passages in Einstein's writings and correspondence that show that he was also unhappy about the indeterminism on its own.

${ }^{11}$ See e.g. Hoefer (2011).

${ }^{12}$ In one common form, the PP simply says that a rational agent, who knows that the objective chance of $A$ is $x$ and has no other/better information about whether or not $A$ will obtain, should set her subjective credence for A equal to $x$ also. See Lewis (1986).

${ }^{13} \mathrm{Or}$ in more Bayesian terms: our subjective credence in the proposition that the objective chance is nearly 0.343 increases, and eventually (if we have "reasonable" priors) approaches unity. 
controversial whether there is any way to argue that PP is justified, for primitive or irreducible (alleged-)probability statements. For this reason, some philosophers (e.g. Lewis (1994)) defend reductive accounts of objective probability and/or probabilistic physical laws. Reductionist approaches will typically deny the possibility of stochastic fundamental laws (e.g. Hoefer (2011)), or demote laws themselves to mere regularities without intrinsic modal force (e.g. Humean best-system approaches such as Lewis (1994) and Loewer (2004)).

When it comes to physical probability, which can be thought of as a species of modality, the same dialectic can arise between those who favor mathematical stochastic laws as primary, and those who instead favor notions such as partial cause, probabilistic disposition, or "chance propensity". The concerns raised above about the semantic content and epistemology of stochastic laws equally affect the latter notions, when they are taken as irreducible primitives.

\subsection{Recovery of the manifest world}

One part of the ambitions encoded in the IV is the idea that physical science can comprehend and account for all features of the external physical world that we all experience. And since at least Eddington's famous discussion of his "two tables" - one the table of everyday experience (solid, continuous, unmoving, impenetrable, colored, etc.), the other the table as apparently described by modern science (a lattice of highly dense points in constant vibratory motion, with much empty space between the atoms or molecules, with no intrinsic color, easily penetrated by many physical things) - an important goal of philosophy of the physical sciences has been to reconcile the world of common sense and daily experience with the world as described by the sciences. This is important in part because we desire to have an overall consistent set of beliefs about the world. But it also has an epistemological side: if the description of the world given by one or more physical theories appears to radically misdescribe the world in certain special ways, then that theory or theories may be held to be epistemically selfundermining: if we took the theory to be true, we would have to doubt the correctness of the very experiences (of scientists, in laboratories and observatories) that is the only basis for believing the theory in the first place. As we will see, this is a particularly acute concern when it comes to quantum physics.

Space does not permit a detailed exploration of the many issues that can be raised concerning the tension between the manifest image and the scientific image of the physical world; here we offer some brief introductions and references.

Flowing time and arrow of time, apparently in tension with fundamental physics

An essential element of our experience of the world is the fact that time seems to pass or flow. This involves the inexorable movement of events that have already occurred further and further into the past, relative to the moment we call now; the equally inexorable approach of future events such as next Monday morning's commute; and the ever-changing nature of the now, or the 'present'. Relatedly, but differently, our experience is full of phenomena that distinguish the past $\rightarrow$ future direction in time from the future $\rightarrow$ past direction. Memory gives us only information about the past, and knowledge of future events - if we have any - is hard to come by and different 
in character. Ice cubes melt in warm drinks, leaving a cooler mix, but we never see a cool mix spontaneously turn into a warm drink with an ice cube floating in it. Waves of water or light frequently diverge with circular symmetry from a point-like source, but we never see such waves converging from far away to a point. And so forth.

The problem is that both time's flow, and time-asymmetry, appear to be absent from fundamental physics. ${ }^{14}$ Some asymmetric phenomena such as the melting of ice cubes can perhaps be handled by imposing a time-asymmetric constraint on initial conditions, e.g. the Boltzmannian statistical mechanics postulate that the universe began in an extremely low-entropy macro state. But such additional postulates are foreign to the fundamental dynamics and their justification is a matter of controversy (see Albert 2003).

In the case of time's flow and the related notion of 'now' or 'the present': not only are these notions absent from physical theories, but relativity theory (Special and General) appear to be incompatible with any objective 'now' or 'present'. In Special Relativity there is no unique way of "foliating" spacetime into slices of space-at-a-time; rather, each inertial reference frame comes with its own way of slicing up spacetime into spaces-at-times. In so far as our manifest notion of the 'now' moving into the future requires the now to be universal, SR renders it perspectival and non-unique. And this situation does not change in any important way in General Relativity. Some philosophers argue that we can be content with a 'now' that is not spatially extended (or not very far-extended). But even if this is accepted, it remains the case that neither SR nor GR contains anything corresponding to the movement of the 'now'; both seem to present spacetime as a 4-d block in which past, present and future are as indifferently equally real as the left and right sides of your kitchen. Despite this, some philosophers argue that the notion of time's passing is both unproblematic and straightforwardly compatible with physics. ${ }^{15}$

Measurement problem of QM: reconciling QM with a determinate (single) macroscopic world It is well known that quantum theory involves a sort of paradox, for which there is as yet no agreed-upon resolution, called the 'measurement problem'. One way to view the problem is this. Quantum mechanics describes physical systems with mathematical functions (wavefunctions) that evolve deterministically over time according to the Schrödinger equation. In certain circumstances we can create, small systems have wavefunctions that are called 'superposition states' with respect to some property (position, spin, momentum, energy etc.), in which the system may not be said to have any definite value of the property in question. When such systems are subjected to measurement, the linearity of the Schrödinger equation entails that the (macroscopic) measurement-indication system should enter into a superposition as well - e.g., having neither the property of simply indicating outcome $x$ nor outcome $y$, but some sort of fusion of both outcomes. But we apparently never see macroscopic systems in

\footnotetext{
${ }^{14}$ An exception to this claim is the t-symmetry violation in parity-non-conserving quantum events such as neutral kaon decay. But such elusive sub-atomic events are not responsible for the overt macro-level time asymmetries just listed.

${ }^{15}$ See, e.g., Maudlin (2002) and Norton (2010). For a defense of a spatially-restricted objective 'now' compatible with relativity theory, see Savitt (2009).
} 
superposition states; instead the measurement yields a single determinate outcome, e.g. $x$.

Much of the effort devoted by physicists and philosophers to "interpreting" quantum theory over the last 90 years has been directed at resolving the measurement problem. Various solutions are available. The standard "textbook recipe" approach simply denies that macroscopic measurement systems get into superposition states: a single outcome occurs, whose probability is given by the Born Rule, and the wavefunction of the measured system (if it continues to exist) 'collapses' into a non-superposition state for the measured property. But this makes the physical theory either inconsistent or incomplete (lacking a precise account of under what circumstances a collapse occurs). On the opposite extreme, the Everett interpretation bites the bullet and asserts that no collapse occurs, macroscopic systems do get into superpositions, and we do "see" them - or rather, we ourselves branch into a superposition state with two (or more) copies of our bodies, one seeing result $x$ and another seeing $y$, etc. This view entails that all possible outcomes do in fact occur in all measurements (and also in many non-measurement situations), which creates a serious interpretive puzzle about probability: what meaning can the Born Rule, i.e. the probabilistic predictions of QM, have? Here we see a danger of epistemic self-undermining looming. Quantum theory is trusted by scientists on the basis of experiments having statistical outcomes matching the probabilistic predictions of the theory very precisely. If an interpretation of the theory makes it difficult to understand those probabilistic predictions, then the interpretation undercuts the evidential basis of the theory. ${ }^{16}$

In between the brute collapse account of the standard recipe and the Everett view there are other approaches to understanding quantum theory that resolve the measurement problem in diverse ways, sometimes by modifying the theory in significant ways. Two examples are the spontaneous collapse theory of Ghirardi, Rimini and Weber (GRW), and Bohmian mechanics, alternative theories based on modifying standard non-relativistic quantum theory. While these alternatives arguably resolve the measurement problem and thereby restore the connection between the theory's predictions and the determinate macroscopic world in one respect, there remain further tensions to resolve, as we will now briefly see.

4-D space-time from quantum theory Non-relativistic quantum theory describes systems - e.g., a carbon atom - with a wavefunction, as we noted above. The wavefunction is not a mathematical object defined as a field in ordinary 3-D space, or 4-D spacetime; instead it is defined in a much higher-dimensional space known as "configuration space", which has $3 \mathrm{~N}$ dimensions, where $\mathrm{N}$ is the number of quantum particles being described. What is the connection between such a mathematical object and the 4-D spacetime (or 3-D spaces existing as time passes ...) we normally think of ourselves as inhabiting? Wavefunctions - or quantum states more generally, constructed in highor infinite-dimensional Hilbert spaces - are essential to the description of physical systems in quantum theory. To treat them as mere instruments with no direct representational significance is always possible, but then this leaves the theory itself as a

\footnotetext{
${ }^{16}$ See Wallace (2012) for extensive discussion of the Everett interpretation, the probability problem and the related epistemic concern of self-undermining, as well as Greaves and Myrvold (2010).
} 
mere instrument, not a description of what reality itself is like, or made of. The alternative of reifying in some physical sense configuration space(s) or Hilbert space(s), however, may seem equally unpalatable. If we take such a space or spaces as part of the fundamental ontology and treat the 3-D space of experience as illusory or merely an (effectively) emergent structure, then we give ourselves an extremely difficult task: showing how our experience of a 3-D world emerges from the high-dimensional underlying quantum reality. And if we postulate that both high-dimensional spaces and 3-D space are real and fundamental, then we give ourselves a problem similar to that which faced Cartesian dualists: giving an account of how the two distinct components of reality can connect, coordinate, and/or influence one another. ${ }^{17}$

This problem of the fundamental dimensionality of physical reality is one currently being much discussed by philosophers of physics (see, e.g., the essays in Ney and Albert 2013). One approach that is being explored is to set aside non-relativistic quantum theory and restrict oneself to quantum field theories, which can (perhaps) be interpreted as only postulating fields existing in 4-D spacetime. ${ }^{18}$

\subsection{Sufficiency of Fundamental Theories}

We now turn to a line of argument challenging the final two commitments of the IV, which characterize a version of reductionism combined with optimism that a "final theory" will ultimately ground physical explanations. Critics of reductionism have recently emphasized the explanatory autonomy and significance of supposedly less fundamental theories, which typically employ theoretical concepts that are not obviously reducible to those of the more fundamental theory. Debates regarding these issues suffer from different formulations of the key concepts - there are a number of different characterizations of "reduction" vs. "emergence," as well as "explanation," on the market (see, e.g, the essays in Bedau and Humphreys 2008). Although we cannot go into depth here, the clarifications of the IV are meant to distinguish it from other versions of reductionism that we regard as clearly too strong. For example, the IV is meant to be compatible with theoretical kinds introduced at the level of the less fundamental theory $T_{i}$ that do not directly map onto those in the more fundamental theory $T_{f}$, as illustrated by multiply realized functional kinds such as temperature. Arguments in favor of emergence based on the novelty of theoretical kinds used by $T_{i}$ are thus not decisive against the IV. Yet the position does still have teeth, in the sense of ruling out stronger senses of emergence, which would be established if there are phenomena successfully described by $T_{i}$ that cannot be accounted for in terms of the ontology and laws of $T_{f}$. Recent philosophical debates regarding the viability of even this modest sense of reductionism have been inspired by foundational studies of a wide range of topics, and more careful assessment of inter-theory relations, in the physical sciences.

\footnotetext{
${ }^{17}$ The obvious option, perhaps, is to demote the quantum state itself to the status of a mere predictive mathematical instrument, not something that directly represents any structure existing in reality. If one takes this route, then there is no pressure to regard physical reality as having more than the familiar 4 spatial dimensions. But then one gives up on the scientific realism part of the IV expressed in the Ontology commitment.

${ }^{18}$ See Myrvold (2015) and Wallace \& Timpson (2010) for examples.
} 
More fundamental theories are clearly not the source of explanations in the sense of providing a complete, computationally tractable account of all phenomena within their domain. Theories ranging from physical chemistry to condensed matter physics employ semi-empirical methods to determine features of the systems being studied. For example, the frequency of the specific hyperfine transitions in cessium-133 atoms used since 1967 to define the second can, in principle, be calculated within quantum theory. But in fact the value of this frequency is determined experimentally; theory supports the identification of this frequency as a useful invariant quantity, but calculations are nowhere near determining the value of this frequency with sufficient precision. In continuum mechanics, the constitutive equations characterizing how a specific type of material responds to strains are motivated phenomenologically rather than derived. The widespread use of such semi-empirical methods reflects the need to supplement the fundamental theory with empirical inputs in applications. There are a variety of other more intriguing cases in which successful applications apparently require appeal to concepts and mathematical structure of the less fundamental theory. Batterman (2002) discusses, for example, the explanation of universal patterns of fringe spacing and intensities of light observed in a variety of cases, such as dark fringes and supernumeray bows observed in rainbows. A theory called catastrophe optics provides successful explanations of these patterns, yet it appeals to mathematical structures of the less fundamental theory (the caustics of ray optics). These aspects of scientific practice challenge any version of reductionism which implies, as Anderson (1972) put it, "the ability to start from those [fundamental] laws and reconstruct the universe." The IV as we formulated it above is not committed to such an implication.

Successful explanations offered by a less fundamental theory $T_{i}$ that are incompatible with the ontology and laws of $\mathrm{T}_{\mathrm{f}}$ would, however, directly challenge the IV, as illustrated by two cases discussed recently by philosophers. First, many systems in classical mechanics are chaotic, in the sense that the trajectories for nearby points in phase space diverge rapidly (exponentially). In the more fundamental theory, quantum mechanics, the unitarity of the dynamics rules out the divergence of trajectories that is the defining feature of classical chaos. This leads to a quite striking contrast between classical and quantum dynamics. Insofar as the exponential divergence of trajectories is crucial to the successful application of classical mechanics, we have a case where the fundamental theory cannot account for the less fundamental theory's success. ${ }^{19}$ Second, thermodynamics (the less fundamental theory in this case) describes phase transitions between distinct phases of matter, such as that between a liquid and gaseous phase of water as the pressure or temperature is slowly changed, in terms of a discontinuity in a thermodynamic quantity. This discontinuity corresponds to a singularity in the partition function in the (more fundamental) statistical mechanical description of the system. Yet the partition function for any system of finite particles is analytic; singularities only arise in the thermodynamic limit, as $\mathrm{N}, \mathrm{V} \rightarrow \infty$ with $\frac{N}{V}=\rho$ (where $N$ is the number of particles and $V$ is the volume). The more fundamental theory hence apparently fails to recover the success of thermodynamics, since the phase transitions occur only in unphysical, idealized systems with an infinite num-

\footnotetext{
${ }^{19}$ There is a large technical literature on this topic; Belot and Earman (1997) and Bokulich (2008) survey some of the technical results, and provide an entry point to the philosophical issues.
} 
ber of particles, rather than in the finite systems of our experience. In both cases, the success of $T_{i}$ depends upon novel features supposedly rendered otiose by $T_{f}$.

These are extremely interesting challenges to the IV, but there are (at least) two responses to cases like these open to defenders of the IV. The first challenges the claim that $T_{i}$ 's success actually supports aspects of the theory that are novel with respect to $T_{f}$. In general we do not expect $T_{f}$ to recover the exact structure of $T_{i}-$ which, after all, has been rejected because it misrepresents some aspects of nature. IV can be preserved if it is possible to costruct a simulacrum of $T_{i}$ 's successful results in $T_{f}$ 's language. Callender (2001) pursues this line of thought with regard to the relationship between statistical mechanics and thermodynamics: we should avoid the error of "taking thermodynamics too seriously," namely of requiring that statistical mechanics reproduces thermodynamics exactly rather than being satisfied with an approximation. In the case of phase transitions, a defense of this line of thought requires showing how mathematical treatments of finite systems approximate various results obtained in the thermodynamic limit. ${ }^{20}$

A second line of response considers the nature of the mathematics used by $T_{i}$ and how it should be interpreted. Belot (2005), for example, argues in reply to Batterman (2002) that the mathematics of wave optics can be understood without appeal to $T_{i}$. The caustics and other structures needed to understand phenomena such as univeral properties of rainbows (as mentioned above) can, in some sense, be discerned within solutions to the wave equations. The debate then turns on the role of the concepts of $\mathrm{T}_{i}$ in interpreting the relevant mathematics; Batterman (2005b) maintains, in response to Belot, that the concepts of ray optics are needed to understand the singular limit of the wave theory. A related line of thought regards the mathematical structures of $T_{i}$ as having merely instrumental utility. One can evade the apparent conflict between the applied mathematics pressed into service in these cases and a reductionist thesis such as IV by denying that the mathematics has any representational significance. For such an instrumentalist approach, although $T_{i}$ may provide a valuable inferential pathway to understanding features of $\mathrm{T}_{\mathrm{f}}$, one need not be troubled if the views along the path differ from those at the destination.

We close by raising a different concern: how would a final theory compare to existing physical theories? One obvious contrast concerns the (often implicit) domains of applicability of current theories. While we expect all current candidates for a fundamental theory to break down at scales where quantum and gravitational effects have to be combined (such as at the Planck scale), presumably the final theory will be truly universal. This may seem like a minimal contrast: we can take existing theories as giving global descriptions of possible worlds distinct from our own, but similar within some domains - e.g. general relativity describes a world free from quantum effects, which may capture many larger-scale features of our own world when gravity is the dominant force. This will not work for philosophers who reject the idea that one can delimit the possible worlds allowed by a theory without considering applications (such as Ruetsche (2011), discussed briefly in §1.3). On Wilson (2006)'s account, the success of classical mechanics depends on what he calls its "facade"-like structure.

\footnotetext{
${ }^{20}$ See Batterman (2005a), Butterfield (2011), Kadanoff (2013), and Menon and Callender (2013) for an entry point into these debates.
} 
Rather than a single axiomatized theory, classical mechanics should be viewed as a collection of different approaches, which include different tools for modeling physical systems. Models succeed in giving detailed descriptions of physical systems in part by restricting consideration to a particular length or time scale, and making a variety of assumptions appropriate for that setting. Wilson describes the resulting overall structure as a facade: the locally applicable models may appear complete, but in fact have implict domain restrictions; attempting to provide a complete description leads to jumping over to a different facade, based on a distinct approach. ${ }^{21}$ The important point for our purposes is that the success of a theory facade does not imply that there is a "globally consistent possible world" described by classical mechanics; instead, we have at best, a patchwork of overlapping local models that cover natural phenomena in much the same way as a set of projections in an atlas cover the globe. ${ }^{22}$ The final theory envisioned by the IV goes well beyond the natural claim that there is a single "way the world is" to assert that there is a single theory that adequately reflects it, without resorting to a facade-like structure.

${ }^{21} \mathrm{He}$ describes, for example, the various ways in which one might describe the physics of billiard ball collisions (see Wilson 2006, Chapter 4.vi). One might start with a description of rigid body collisions with results dictated by conservation principles. But this simple description fails to describe energy loss during the collision, distortion of the impacting bodies, and a variety of other effects. Wilson argues that more sophisticated models of collisions based on, e.g., continuum mechanics cannot be seen as straightforwardly "completing" or "augmenting" the simple account, since these models include physical and mathematical assumptions incompatible with the earlier approach.

${ }^{22}$ Wilson describes the facade-like structure of classical mechanics as a consequence of the way classical mechanics "sits on top of" quantum mechanics, and he does not argue (as far as I am aware) for the more general claim that all theories must have a facade-like structure. But an advocate of a more general facade thesis could certainly build on Wilson's discussion of concept use. 


\section{References}

Albert, D.Z. (2003). Time and Chance. Harvard University Press.

Anderson, Philip W (1972). More is different. Science, 177(4047), 393-396.

Batterman, Robert W (2002). The devil in the details: Asymptotic reasoning in explanation, reduction, and emergence. Oxford University Press, Oxford.

Batterman, Robert W (2005a). Critical phenomena and breaking drops: Infinite idealizations in physics. Studies In History and Philosophy of Science Part B: Studies In History and Philosophy of Modern Physics, 36(2), 225-244.

Batterman, Robert W (2005b). Response to Belot's "Whose Devil? Which Details?". Philosophy of Science, 72, 154-163.

Bedau, Mark A and Humphreys, Paul Ed (2008). Emergence: Contemporary readings in philosophy and science. MIT press.

Belot, Gordon (2005). Whose devil? which details? Philosophy of Science, 72(1), 128-153.

Belot, Gordon and Earman, John (1997). Chaos out of order: Quantum mechanics, the correspondence principle and chaos. Studies in History and Philosophy of Science Part B: Studies in History and Philosophy of Modern Physics, 28(2), 147-182.

Bokulich, Alisa (2008). Reexamining the Quantum-Classical Relation. Cambridge University Press Cambridge.

Butterfield, Jeremy (2011). Less is different: emergence and reduction reconciled. Foundations of Physics, 41(6), 1065-1135.

Callender, Craig (2001). Taking thermodynamics too seriously. Studies In History and Philosophy of Science Part B: Studies In History and Philosophy of Modern Physics, 32(4), 539-553.

Cartwright, N. (1983). How the Laws of Physics Lie. Clarendon paperbacks. Clarendon Press.

Colbeck, Roger and Renner, Renato (2012). Free randomness can be amplified. $\mathrm{Na}$ ture Physics, 8(6), 450-453.

Filomeno, Aldo (2014). On the Possibility of Stable Regularities Without Fundamental Laws. Ph.D. thesis, Autonomous University of Barcelona.

Fine, A. (1986). The Shaky Game: Einstein, Realism, and the Quantum Theory. Science and its conceptual foundations. University of Chicago Press.

Frisch, Mathias (2009a). Causality and dispersion: A reply to john norton. British Journal for the Philosophy of Science, 60(3), 487-495.

Frisch, Mathias (2009b). 'the most sacred tenet'? causal reasoning in physics. British Journal for the Philosophy of Science, 60(3), 459-474.

Frisch, Mathias (2012). No place for causes? causal skepticism in physics. European Journal for Philosophy of Physics, 2(3), 313-336.

Froggatt, C.D. and Nielsen, H.B. (1991). Origin of Symmetries. World Scientific. 
Greaves, Hilary and Myrvold, Wayne (2010). Everett and evidence. In Many Worlds (ed. S. Saunders, J. Barrett, and A. Kent), pp. 264-304. Oxford University Press.

Hoefer, Carl (2003). For Fundamentalism. Philosophy of Science, 70(5), 1401-1412. Hoefer, Carl (2011). Time and chance propensities. The Oxford Handbook of Philosophy of Time, 68-90.

Kadanoff, Leo P (2013). Theories of matter: infinities and renormalization. In The Oxford Handbook of Philosophy of Physics (ed. R. W. Batterman), pp. 141-188. Oxford University Press, Oxford.

Lewis, David (1986). In Philosophical Papers: Vol. II, pp. 83-113. Oxford University Press.

Lewis, David (1994). Humean supervenience debugged. Mind, 103(412), 473-490.

Loewer, Barry (2004a). In Readings on Laws of Nature (ed. J. Carroll), p. 176206. Pittsburgh University Press.

Loewer, Barry (2004b). David lewis's humean theory of objective chance. Philosophy of Science, 71(5), 1115-25.

Malament, David B (2007). Classical relativity theory. Handbook of the philosophy of science. Philosophy of physics. Part A. Amsterdam: Elsevier, 229-275.

Maudlin, Tim (2002). Remarks on the passing of time. Proceedings of the Aristotelian Society, 102, pp. 259-274.

Menon, Tarun and Callender, Craig (2013). Ch-ch-changes philosophical questions raised by phase transitions. In The Oxford Handbook of Philosophy of Physics (ed. R. W. Batterman), pp. 189-223. Oxford University Press, Oxford.

Myrvold, Wayne C. (2015). What is a wavefunction? Synthese, 1-28.

Myrvold, Wayne C. (forthcoming). Probabilities in statistical mechanics. In The Oxford Handbook of Probability and Philosophy (ed. C. Hitchcock and A. Hájek). Oxford University Press.

Ney, Alyssa and Albert, David Z (2013). The wave function: Essays on the metaphysics of quantum mechanics. Oxford University Press.

Norton, John D. (2007). Causation as folk science. In Philosophers' Imprint (ed. H. Price and R. Corry), Volume 3. Oxford University Press.

Norton, John D. (2009). Is there an independent principle of causality in physics? British Journal for the Philosophy of Science, 60(3), 475-486.

Norton, John D. (2010). Time really passes. Humana.Mente (online).

Pironio, S., Acín, A. et al. (2010). Random numbers certified by bells theorem. Nature, 464(7291), 1021-1024.

Ruetsche, Laura (2011). Interpreting quantum theories: The art of the possible. Oxford University Press, Oxford.

Russell, Bertrand (1912). On the notion of cause. In Proceedings of the Aristotelian society, pp. 1-26. JSTOR.

Savitt, StevenF. (2009). The transient nows. In Quantum Reality, Relativistic Causality, and Closing the Epistemic Circle, Volume 73, The Western Ontario Series in Philosophy of Science, pp. 349-362. Springer Netherlands.

Smith, Sheldon (2002). Violated laws, ceteris paribus clauses, and capacities. Synthese, 130(2), 235-264.

Smith, Sheldon R. (2013). Causation in classical mechanics. In The Oxford Handbook 
of Philosophy of Physics (ed. R. W. Batterman), pp. 107-140. Oxford University Press, Oxford.

Strevens, Michael (2003). Bigger Than Chaos: Understanding Complexity Through Probability. Number 4. Harvard University Press.

Strevens, Michael (2011). Probability out of determinism. In Probabilities in Physics (ed. C. Beisbart and S. Hartmann), pp. 339-364. Oxford University Press.

Unger, R.M. and Smolin, L. (2014). The Singular Universe and the Reality of Time. Cambridge University Press.

van Fraassen, Bas (1989). Laws and Symmetry. Oxford University Press, Oxford.

Wallace, D. (2012). The Emergent Multiverse: Quantum Theory According to the Everett Interpretation. OUP Oxford.

Wallace, David and Timpson, Christopher Gordon (2010). Quantum mechanics on spacetime i: Spacetime state realism. British Journal for the Philosophy of Science, 61(4), 697-727.

Wheeler, J.A. (1982). Physics and Austerity: Law Without Law : Working Paper. Center for Theoretical Physics, University of Texas.

Wilson, Mark (1985). What is this thing called pain?-the philosophy of science behind the contemporary debate. Pacific philosophical quarterly, 66(3-4), 227-267.

Wilson, Mark (2006). Wandering significance: an essay on conceptual behavior. Oxford University Press, Oxford.

Woodward, J. (2003). Making Things Happen: A Theory of Causal Explanation. Oxford Studies in the Philosophy of Science. Oxford University Press, USA. 